\title{
Euler-Poincaré Characteristic and Polynomial Representations of Iwahori-Hecke Algebras
}

\author{
By \\ Gérard Duchamp *, Daniel Krob**, Alain LAScoux **, \\ Bernard LECLERC ***, Thomas SCHARF **** \\ and Jean-Yves THIBON ****
}

\begin{abstract}
The Hecke algebras of type $A_{n}$ admit faithful representations by symmetrization operators acting on polynomial rings. These operators are related to the geometry of flag manifolds and in particular to a generalized Euler-Poincaré characteristic defined by Hirzebruch. They provide $q$-idempotents, togetherwith a simple way to describe the irreducible representations of the Hecke algebra. The link with Kazhdan-Lusztig representations is discussed. We specially detail the case of hook representations, and as an application, we investigate the hamiltonian of a quantum spin chain with $U_{q}(\mathfrak{s u}(1 / 1))$ symmetry.
\end{abstract}

\section{§1. Introduction}

The Iwahori-Hecke algebra $\mathscr{H}_{n}$ associated to the symmetric group $\mathfrak{S}_{n}$ admits a faithful representation as an algebra of operators on the ring of polynomials $K$ $[\mathbf{A}], \mathbf{A}=\left\{a_{1}, \ldots, a_{n}\right\}$ [LS 82-87].

As a matter of fact, such operators have already been used to describe flag manifolds : Hirzebruch [Hi1] has defined a " $\chi_{y}$-characteristic" which coincide with the operators that we use in the case of maximal permutations of a Young subgroup, when extended to the case of a relative flag variety. In particular, for simple transpositions the operators correspond to the characteristic of a projective

Communicated by T. Miwa, December 7, 1993.

1991 Mathematics Subject Classifications : 05E10, 20C30, 14M15, $20 \mathrm{G} 45$.

* L. I. R., Université de Rouen, Place E. Blondel, B. P. 118, 76134 Mont-Saint-Aignan Cedex, France

** L. I. T. P., Université Paris 7, 2 place Jussieu, 75251 Paris Cedex 05, France

*** Institut Gaspard Monge, Université de Marne-la-Vallée, 2 rue de la Butte-Verte, 93166 Noisy-leGrand Cedex, France

**** Lehrstuhl II f. Math., Universität Bayreuth, Postfach 101251, D-W-8580 Bayreuth, Germany 
line bundle. However, the simple operators do not satisfy Moore-Coxeter relations, but Yang-Baxter ones (Section 3). We give in Theorem 3.1 several expressions of the operators corresponding to a maximal permutation.

Restricting the action of the Hecke algebra to weight spaces, we recover one of the usual descriptions of its representations (Section 4).

We also obtain $q$-idempotents in Section 5, and deduce from them a $q$-analog of Specht representation, as an orbit of a product of $q$-Vandermonde functions. We moreover show that the decomposition of general elements into a standard basis can be obtained from a $q$-analog of a triangular matrix due to Rutherford (Proposition 5.3).

Different constructions of the irreducible representations are exhibited in the case corresponding to hook partitions. We show that these representations are essentially described in terms of certain graphs, which turn out to be identical with those of Kazhdan-Lusztig (Section 7).

This interpretation is then applied to the diagonalization of the hamiltonian of a quantum spin chain having the quantum superalgebra $U_{q}(\mathfrak{s u}(1 / 1))$ as symmetry algebra.

\section{§ 2. Symmetrizing Operators}

Our fundamental tool will be Newton's divided differences and deformations of them called "symmetrizing operators". All operators act on their left.

Denote by $\sigma_{i}, 1 \leq i \leq n-1$, the simple transposition exchanging $a_{\imath}$ and $a_{i+1}$. The Newton divided difference associated to the pair $a_{i}, a_{i+1}$ is the operator on $K[\mathbf{A}]$

$$
\partial_{i}: f \rightarrow\left(f-f^{\sigma_{i}}\right) /\left(a_{i}-a_{i+1}\right) .
$$

The $\partial_{i}$ satisfy the relations

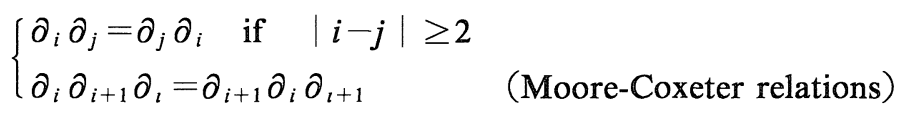

together with

$$
\partial_{i}^{2}=0
$$

More general operators are :

$$
D_{i}(p, q, r): f \rightarrow \frac{\left(p+(q-1) a_{i}\right) f-\left(p+(q-1) a_{t+1}\right) f^{\sigma_{i}}}{a_{t}-a_{\imath+1}}+r f^{\sigma_{i}}
$$

which satisfy Coxeter relations and 


$$
D_{i}^{2}=(q-1) D_{i}+r(q+r-1)
$$

In [LS87] one finds more general five-parameters symmetrizing operators having similar properties.

Coxeter relations imply that for any $\mu \in S_{n}$, there exists a well defined operator $D_{\mu}$ which is equal to the product of the simple operators $D_{i}$ corresponding to any reduced decomposition of $\mu$. In particular, one has for any permutation $\mu$ a corresponding divided difference $\partial_{\mu}[\mathrm{BGG}]$, [Dem $]$.

One notices that the operators $T_{i}:=D_{i}(0, q, 1)$ satisfy the usual Hecke relation

$$
\left(T_{i}+1\right)\left(T_{i}-q\right)=0,
$$

and thus that the $T_{i}$ generate a representation of $\mathscr{H}_{n}$. The $T_{\mu}=D_{\mu}(0, q, 1)$ constitute a linear basis of $\mathscr{H}_{n}$.

This representation is faithful, so that we can characterize elements of $\mathscr{H}_{n}$ by considering their explicit action on $K[\mathbf{A}]$.

Since the operators $D_{l}$ admit symmetric functions as scalars, we can use the structure of $K[\boldsymbol{A}]$ as a free $\mathfrak{S}_{\mathfrak{y m}}(\mathbb{A})$ module of $\operatorname{rank} n$ ! (where $\mathfrak{S}_{\mathfrak{y m}}(\mathbf{A})$ denotes the ring of symmetric polynomials of $K[\mathbf{A}]$ ).

It is therefore sufficient to restrict the action to an appropriate basis. For example, relation (2.1) requires to be checked on $K\left[a_{l}, a_{i+1}\right]$, which is a free $\mathfrak{S n m}_{\mathfrak{y}}$ $\left(a_{l}, a_{l+1}\right)$-module with basis $\left\{1, a_{i}\right\}$. Now, $D_{i}$ sends 1 to $q-1+r$ and $a_{i}$ to $p+$ $(q-1)\left(a_{\imath}+a_{i+1}\right)+r a_{i+1}$, and thus one has relation (2.1).

Given a complex compact manifold $\mathscr{M}$, and any analytic vector bundle $V$ on it, Hirzebruch $[\mathrm{Hi1}]$ defined the $\chi_{y}$-characteristic of $V$ to be

$$
\chi_{y}(V):=\sum_{i, j} y^{j}(-1)^{i} \operatorname{dim} H^{i}\left(\mathscr{M}, V \otimes \Omega^{\jmath}\right),
$$

where $\Omega^{J}$ is the $j$-th exterior power of the cotangent bundle $\Omega^{1}$ of $\mathscr{M}$.

The three special cases $y=0,1$ or -1 are of particular importance in geometry, when $V$ is the trivial line bundle (denoted 1): $\chi_{-1}(1)$ is the ordinary EulerPoincaré characteristic. If $\mathscr{M}$ is a compact Kähler manifold, then $\chi_{1}(1)$ is the signature of $\mathscr{M}$ and $\chi_{0}(1)$ is its arithmetic genus (see [Hi2]).

As shown by Grothendieck, one should work with relative varieties $\mathscr{M}^{p} \rightarrow \mathscr{B}$ and their associated Grothendieck rings $K_{0}(\mathscr{M}) \stackrel{p^{\prime}}{\rightarrow} K_{0}(\mathscr{B})$ of classes of vector bundles.

The $\chi_{y}$-characteristic becomes now the following morphism (called relative $\chi_{y}$-characteristic, cf. [Las]) :

$$
K_{0}(\mathscr{M}) \ni[V] \rightarrow \chi_{y}(V):=\sum_{i, j} y^{j}(-1)^{\imath} \mathscr{R}^{i} p_{*}\left(V \otimes \Omega_{\mathscr{M} / \mathscr{B}}^{j}\right) \in K_{0}(\mathscr{B})
$$

We shall detail the case of flag manifolds. Let $\mathscr{B}$ be a complex manifold, $V$ a 
vector bundle of rank $n$ on it, $\mathscr{F}(V)$ the associated flag bundle. Let $L_{1} \ldots, L_{n}$ be the tautological line bundles on $\mathscr{F}(V)$ and $a_{1}, \ldots, a_{n}$ their respective classes in $K_{0}(\mathscr{F}(V))$. The Grothendieck ring of $\mathscr{F}(V)$ is the quotient of the ring $K_{0}(\mathscr{B})$ $\left[a_{1}, \ldots, a_{n}\right]$ by the ideal $\mathscr{I}$ generated by the graded relation $\Pi\left(1+a_{i}\right)=\sum\left[\Lambda^{i} V\right]$.

The associated morphism $p$, is

$$
f \rightarrow \sum_{\mu \in \mathbb{E}_{n}}\left[f / \prod_{J>1}\left(1-a_{j} / a_{i}\right)\right]^{\mu},
$$

where $g^{\mu}$ denotes the image of a function of $a_{1}, \ldots, a_{n}$ under a permutation $\mu$.

The class of the relative cotangent bundle $\Omega^{1}$ is equal to $\Sigma_{n \geq_{j}>i \geq 1} a_{j} / a_{i}$ and thus $\lambda_{-1 / q}\left(\Omega^{1}\right):=\Sigma(-q)^{-i} \wedge^{i} \Omega^{1}$ is equal to $\Pi_{1 \leq i<j \leq n}\left(1-q^{-1} a_{j} / a_{l}\right)$.

Finally, in the case of a relative flag manifold, the $\chi_{y}$-characteristic, for $y=$ $1 / q$, is the morphism

$$
K_{0}(\mathscr{B})\left[a_{1}, \ldots, a_{n}\right] / \mathscr{I} \ni f \rightarrow \sum_{\mu \in \mathscr{E}_{n}}\left[f \frac{\prod_{j>i}\left(a_{i}-a_{j} / q\right)}{\prod_{j>i}\left(a_{i}-a_{j}\right)}\right]^{\mu}
$$

In the particular case $n=2$ (case of a relative projective line) Hirzebruch's characteristic is equal to the operator $f \rightarrow\left(a_{1}-a_{2} / q\right) \partial_{1}$. Let us rather use the operators (acting on their left)

$$
\square_{i}:=\left(q a_{i}-a_{i+1}\right) \partial_{i}=T_{i}+1
$$

We shall see in the sequel that $\square_{1}\left(\square_{2}-\frac{q}{1+q}\right) \square_{1}$ is also equal to Hirzebruch's characteristic for a flag variety with $n=3$, i. e. that one has

$$
\square_{1}\left(\square_{2}-\frac{q}{1+q}\right) \square_{1}=\left(q a_{1}-a_{2}\right)\left(q a_{1}-a_{3}\right)\left(q a_{2}-a_{3}\right) \partial_{321} .
$$

Symmetrically, one also has operators

$$
\nabla_{i}:=\partial_{i}\left(q a_{i+1}-a_{i}\right)=T_{i}-q
$$

as well as a dual characteristic

$$
\nabla_{\omega}:=\partial_{\omega} \prod_{1 \leq i<j \leq n}\left(q a_{i+1}-a_{i}\right) .
$$

The operators $\square_{i}$ and $\nabla_{i}$ satisfy the relations

$$
\square_{i}^{2}=(q+1) \square_{i}, \quad \nabla_{i}^{2}=-(q+1) \nabla_{i},
$$

which will be used to construct $q$-idempotents (see Section 6). 
However, these operators do not satisfy Moore-Coxeter relations, but Yang-Baxter ones :

$$
\begin{gathered}
\square_{i}\left(\square_{i+1}-\frac{q}{1+q}\right) \square_{i}=\square_{i+1}\left(\square_{i}-\frac{q}{1+q}\right) \square_{i+1}, \\
\nabla_{i}\left(\nabla_{i+1}+\frac{q}{1+q}\right) \nabla_{i}=\nabla_{i+1}\left(\nabla_{i}+\frac{q}{1+q}\right) \nabla_{i+1} .
\end{gathered}
$$

These relations are better understood when extending them to the case of any reduced decomposition, instead of only $\sigma_{i} \sigma_{i+1} \sigma_{i}$.

\section{§ 3. Yang-Baxter Equation}

Let $\sigma_{i} \sigma_{j} \sigma_{h} \ldots \sigma_{k}$ be a reduced decomposition of the permutation $\mu$. This reduced decomposition gives rise to the sequence of transpositions

$$
\tau^{1}:=\sigma_{i}, \quad \tau^{2}:=\left(\sigma_{i}\right) \sigma_{j}\left(\sigma_{i}\right), \quad \tau^{3}:=\left(\sigma_{i} \sigma_{j}\right) \sigma_{h}\left(\sigma_{j} \sigma_{i}\right), \ldots
$$

The total set of transpositions, which corresponds to the set of inversions of $\mu$, does not depend on the choice of the reduced decomposition of $\mu$.

A function $f$ from the set of transpositions to any commutative ring will be called a solution of Yang-Baxter equation if it is such that, for any permutation $\mu$ and for any reduced decomposition of $\mu$, the products

$$
\begin{gathered}
\left(\nabla_{i}+f\left(\tau^{1}\right)\right)\left(\nabla_{j}+f\left(\tau^{2}\right)\right)\left(\nabla_{h}+f\left(\tau^{3}\right)\right) \ldots \\
\left(\square_{\imath}-f\left(\tau^{1}\right)\right)\left(\square_{j}-f\left(\tau^{2}\right)\right)\left(\square_{h}-f\left(\tau^{3}\right)\right) \cdots
\end{gathered}
$$

are operators independent of the choice of the reduced decomposition of $\mu$.

A solution, implicit in [Ro] and used by Cherednik [Che1-2] is, taking an extra set of variables $\left\{x_{1}, \ldots, x_{n}\right\}$ :

$$
f\left(\tau_{i j}\right)=\left(q x_{i}-x_{j}\right) /\left(x_{i}-x_{j}\right)
$$

for the transposition $\tau_{i j}$ of $i, j$.

$[F K 1]$ and [FK2] explicit the links between the usual form of Yang-Baxter equation and the operators $\partial_{i}$ and $a_{t} \partial_{i}$, and their associated polynomials (Schubert polynomials, Grothendieck polynomials).

We shall need only the special case where $\left\{x_{1}, x_{2}, \ldots, x_{n}\right\}=\left\{1, q, q^{2}, \ldots\right.$, $\left.q^{n-1}\right\}$. In that case, $f\left(\tau_{l j}\right)=q \frac{[j-i-1]}{[j-l]}$, writing $[r]$ for the $q$-integer $\left(1-q^{r}\right) /(1-$ $q)$.

For any permutation $\mu$, let us denote $\nabla_{\mu}, \square_{\mu}$ the operators given by this 
solution of Yang-Baxter equation.

For example, $\sigma_{2} \sigma_{3} \sigma_{1} \sigma_{2}$ is a reduced decomposition of $\mu=3412$. The associated sequence of transpositions is $\sigma_{2}=(32), \sigma_{2} \sigma_{3} \sigma_{2}=(42), \sigma_{2} \sigma_{3} \sigma_{1} \sigma_{3} \sigma_{2}=$ (31), $\sigma_{2} \sigma_{3} \sigma_{1} \sigma_{2} \sigma_{1} \sigma_{3} \sigma_{2}=(41)$. The two operators are

$$
\begin{aligned}
& \nabla_{3412}=\nabla_{2}\left(\nabla_{3}+\frac{q}{1+q}\right)\left(\nabla_{1}+\frac{q}{1+q}\right)\left(\nabla_{2}+\frac{q+q^{2}}{1+q+q^{2}}\right), \\
& \square_{3412}=\square_{2}\left(\square_{3}-\frac{q}{1+q}\right)\left(\square_{1}-\frac{q}{1+q}\right)\left(\square_{2}-\frac{q+q^{2}}{1+q+q^{2}}\right) .
\end{aligned}
$$

We are now in position to recover Euler-Poincaré characteristic.

Theorem 3.1. Let $\omega$ be the maximal permutation of $\mathfrak{S}_{n}$. Then

i) $\nabla_{\omega}=\nabla_{1}\left(\left(\nabla_{2}+q \frac{[1]}{[2]}\right) \nabla_{1}\right)\left(\left(\nabla_{3}+q \frac{[2]}{[3]}\right)\left(\nabla_{2}+q \frac{[1]}{[2]}\right) \nabla_{1}\right) \ldots$

$$
\ldots\left(\left(\nabla_{n-1}+q \frac{[n-1]}{[n]}\right) \ldots\left(\nabla_{2}+q \frac{[1]}{[2]}\right) \nabla_{1}\right)=\partial_{\omega} \prod_{l<J}\left(q a_{j}-a_{i}\right)=\sum_{\mu \in \mathbb{E}_{n}}(-q)^{l(\omega \mu)} \mathbb{T}_{\mu}
$$

ii) $\square_{\omega}=\square_{1}\left(\left(\square_{2}-q \frac{[1]}{[2]}\right) \square_{1}\right)\left(\left(\square_{3}-q \frac{[2]}{[3]}\right)\left(\square_{2}-q \frac{[1]}{[2]}\right) \square_{1}\right) \ldots$

$$
\ldots\left(\left(\square_{n-1}-q \frac{[n-1]}{[n]}\right) \ldots\left(\square_{2}-q \frac{[1]}{[2]}\right) \square_{1}\right)=\prod_{i<j}\left(q a_{i}-a_{j}\right) \partial_{\omega}=\sum_{\mu \in \mathbb{E}_{n}} T_{\mu} \text {. }
$$

Proof. Take the basis $X_{\mu}, \mu \in \Im_{n}$ of Schubert polynomials (cf. [LS82]). If there exists some $i$ such that $\sigma_{i}$ leaves invariant $X_{\mu}$, then $X_{\mu}$ is annihilated by $\partial_{i}$ and thus by $\partial_{\omega}$ as well as $\nabla_{\omega}$, because there exists at least one reduced decomposition of $\omega$ beginning by $\sigma_{i}$. The same reasoning is valid for the sum $\Sigma(-q)^{l(\omega \mu)} T_{\mu}$, from which one can extract a left factor $T_{l}-q=\partial_{i}\left(q a_{i+j}-a_{i}\right)$. Therefore, one has only to compute the action of the three operators on the only totally non symmetrical Schubert polynomial, which is $X_{\omega}=a_{1}^{n-1} a_{2}^{n-2} \cdots a_{n-1}$, and this can be done by induction on $n$. Left/right symmetry gives $i i$ ) from $i$ ).

Remarks. 1) Let $\mathbb{I} \in \mathbb{N}$ and $\mathfrak{S}(I)=\mathfrak{S}\left(i_{1}\right) \times \mathfrak{S}\left(i_{2}\right) \times \ldots \times \mathfrak{S}\left(i_{k}\right)$ be the Young subgroup corresponding to $\mathbb{I}$. The preceding theorem extends to the case of the maximal permutation $\omega_{I}$ of $\mathfrak{S}(I)$. For example, for $\mathfrak{S}(3,2)=\mathfrak{S}(3) \times \mathfrak{S}(2)$, one has $\omega_{I}=\left(\begin{array}{lllll}1 & 2 & 3 & 4 & 5 \\ 3 & 2 & 1 & 5 & 4\end{array}\right)$ and

$$
\begin{aligned}
\nabla_{32154} & =\nabla_{2}\left(\nabla_{1}+\frac{q}{1+q}\right) \nabla_{2} \nabla_{4} \\
& =\partial_{32154}\left(\left(q a_{3}-a_{1}\right)\left(q a_{2}-a_{1}\right)\left(q a_{3}-a_{2}\right)\right)\left(q a_{5}-a_{4}\right)
\end{aligned}
$$




$$
=\sum_{\mu \in \mathbb{E}(3,2)}(-q)^{4-l(\mu)} T_{\mu}
$$

2) Geometry provides a fibration of the flag manifold into projective spaces ( $[\mathrm{Hi2}]$ p. 49), and this induces the following factorization, with $\omega^{\prime}=(n-1, \ldots, 1$, n):

$$
\square_{\omega}=\square_{\omega^{\prime}}\left(\prod_{1 \leq 1<n}\left(q a_{i}-a_{n}\right)\right) \partial_{n-1} \ldots \partial_{1}
$$

One has also the factorisation

$$
\square_{\omega}=\square_{\omega^{\prime}}\left(1+T_{n-1}+T_{n-1} T_{n-2}+\ldots T_{n-1} \cdots T_{1}\right),
$$

but the two operators $\Pi_{1 \leq i<n}\left(q a_{i}-a_{n}\right) \partial_{n-1} \cdots \partial_{1}$ and $1+T_{n-1}+T_{n-1} T_{n-2}+\ldots$ $T_{n-1} \cdots T_{1}$ are not equal.

3) The operator $\square_{\omega}$ sends any monomial onto a Hall-Littlewood polynomial (cf. $[\mathrm{Mcd}]$ ). For example with $\mathrm{A}=\left\{a_{1}, a_{2}, a_{3}\right\}$, one has $a_{1}^{4} a_{2}^{2} \square_{321}=q^{6} P_{42}(\mathrm{~A}, 1$ ) $q)$.

\section{§4. Weight Spaces}

Let $\mathbf{A}=\left\{a_{1}, \ldots, a_{n}\right\}$ be an alphabet of cardinal $n$. For every $J=\left(j_{1}, j_{2}, \ldots\right.$ ,$\left.j_{n}\right) \in \mathbf{N}^{n}$, one denotes by $e^{J}$ the monomial

$$
e^{J}:=a_{1}^{j_{1}} a_{\dot{L}^{2}}^{\dot{x}_{2}} \ldots a_{n^{n}}^{j_{n}}
$$

and $\mathscr{V}(J)$ the weight space of all monomials $\left\{e^{J_{\mu}}: \mu \in \mathbb{S}(\mathbf{A})\right\}$, i.e. the space generated by the action of the symmetric group $\subseteq(\mathbf{A})$ on $e^{J}, J \mu$ being the vector $\left(j_{\mu_{1}}, \ldots, j_{\mu_{n}}\right)$.

A weakly decreasing $J$ is called a partition and the corresponding monomial $e^{J}$, a dominant monomial. A weakly increasing $J$ is called a French partition. The socalled natural order on partitions $[\mathrm{JK}]$ induces an order on weight spaces of the same degree $|\boldsymbol{J}|:=j_{1}+\cdots+j_{n}$.

The operators $T, \square, \nabla$ do not preserve weight spaces. Indeed, let $T$ correspond to the pair of letters $a, b$ and let $h<k$ be positive integral numbers. Then one has

$$
\begin{gathered}
a^{h} b^{k} \stackrel{T}{\longrightarrow}(1-q)\left(a^{h+1} b^{k-1}+\cdots+a^{k-1} b^{h+1}\right)+a^{k} b^{h},(h<k-1) \\
a^{h} b^{h+1} \stackrel{T}{\longrightarrow} a^{h+1} b^{h} \\
a^{h} b^{h} \stackrel{T}{\longrightarrow} q a^{h} b^{h} \\
a^{k} b^{h} \stackrel{T}{\longrightarrow} q a^{h} b^{k}+(q-1)\left(a^{h+1} b^{k-1}+\cdots+a^{k-1} b^{h+1}\right)+(q-1) a^{k} b^{h} .
\end{gathered}
$$


Modulo the weight spaces $\mathscr{V}(i j): h<i \leq j<k$, $i+j=h+k$, spaces which are smaller with respect to the order induced from the order on partitions, the action of $T$ is

$$
\begin{gathered}
a^{h} b^{k} \stackrel{T}{\longrightarrow} a^{k} b^{h}, \quad a^{k} b^{h} \stackrel{T}{\longrightarrow} q a^{h} b^{k}+(q-1) a^{k} b^{h} \\
a^{h} b^{h} \stackrel{T}{\longrightarrow} q a^{h} b^{h} .
\end{gathered}
$$

Notice that the action modulo the smaller weight spaces does not depend on the value of $h$ and $k$, but only on the fact that $h<k, h=k$ or $h>k$.

Let $\mathfrak{p}_{J}$ denote the projection from the space of polynomials onto the space $\mathscr{V}$ $(J)$ (i. e. sending to 0 all monomials which do not belong to $\mathscr{V}(J)$ ). Writing $[I]$ instead of $e^{I}$, what we said just above can be formalized in :

Proposition 4. 目 i) Let $I=\left(i_{1}, \ldots, i_{n}\right)$ be any integral vector in $\mathbb{N}^{r}, \lambda_{1}, \ldots$ ,$\lambda_{r}$ rational functions in $q$, and $\mathscr{V}(\mathbb{J})$ is a weight space. Then $\mathfrak{p}_{J}\left(\mathbb{T}_{i_{1}}+\lambda_{1}\right) \cdots\left(\mathbb{T}_{i_{r}}+\right.$ $\left.\lambda_{r}\right) \mathfrak{p}_{J}=\left(\mathfrak{p}_{J}\left(\mathbb{T}_{i_{1}}+\lambda_{1}\right) \mathfrak{p}_{J}\right) \cdots\left(\mathfrak{p}_{J}\left(\mathbb{T}_{i_{r}}+\lambda_{r}\right) \mathfrak{p}_{J}\right)$

ii) The operators:

$$
\left\{\begin{array}{l}
{[\cdots h k \cdots] \longrightarrow[\cdots k h \cdots]} \\
{[\cdots h h \cdots] \longrightarrow q[\cdots h h \cdots]} \\
{[\cdots k h \cdots] \longrightarrow q[\cdots h k \cdots]+(q-1)[\cdots k h \cdots]}
\end{array}\right.
$$

operating on the $r$ and $r+1$ component, with $h<k$, generate a representation of the Hecke algebra which preserves each weight space.

If one prefers, one can write the second point of the proposition :

iii) For any French partition $J$, the weight space is a representation of the Hecke algebra, with the generators $\mathfrak{p}_{J} \mathbb{T}_{i} \mathfrak{p}_{J}$ defined by :

$$
\begin{aligned}
& \left(e^{J \mu}\right) \mathfrak{p}_{J} T_{i} \mathfrak{p}_{J}=e^{J \mu \sigma_{i}} \text { if } l\left(J \mu \sigma_{i}\right)<l(J \mu) \\
& \left(e^{J \mu}\right) \mathfrak{p}_{J} T_{i} \mathfrak{p}_{J}=q e^{J \mu} \text { if } J \mu \sigma_{i}=J \mu \\
& \left(e^{J \mu}\right) \mathfrak{p}_{J} T_{i} \mathfrak{p}_{J}=q e^{J \mu \sigma_{l}}+(q-1) e^{J \mu} \text { if } l\left(J \mu \sigma_{\imath}\right)>l\left(J \mu \sigma_{i}\right) .
\end{aligned}
$$

When $J$ has all its components $j_{1}, \ldots, j_{n}$ different, the weight space $\mathscr{V}(J)$ is the regular representation.

Notice that restricting the action of the Iwahori/Hecke algebra, we have lost the fact that symmetric functions in are scalars for the operators.

Interpreting the 2-component symbols $[h, k]$ as tensor products $e_{h} \otimes e_{k}$ of the basis vectors $e_{i}$ of some $n$-dimensional vector space $V$, we recover from $i i$ ) a familiar solution of the (quantum) Yang-Baxter equation 


$$
R=\sum_{h<k} E_{k h} \otimes E_{h k}+q \sum_{h} E_{h h} \otimes E_{h h}+q \sum_{h<k} E_{h k} \otimes E_{k h}+(q-1) \sum_{h<k} E_{k k} \otimes E_{h h}
$$

where $E_{i j}$ is the matrix with coefficients $\left(E_{\imath j}\right)_{k l}=\delta_{i k} \delta_{j l}$.

\section{§ 5. q-Specht Representations}

In the case of the symmetric group, one has many ways to define irreducible representations. The original method of Young was to associate an idempotent to any Young tableau. The idempotents corresponding to all the tableaux of a given shape are all conjugate, so that Young defined essentially only one idempotent for each shape. Because conjugation is more tricky in a Hecke algebra, one has more $q$-idempotents corresponding to a given shape. Let us just define one of them, following a construction of Martin [Ma2]. Gyoja [Gy] and Dipper and James $[\mathrm{DJ}]$ had also described $q$-idempotents in a sligthly different way.

Let $I$ be a decreasing partition, $J$ be the conjugate partition, this time increasing, and let $\omega^{\prime}$ and $\omega^{\prime \prime}$ the maximal permutations of the two Young subgroups $\mathfrak{S}(I)$ and $\mathfrak{S}(J)$. Then (cf. Martin [Ma2])

Lemma 5. 1. The space $\nabla_{\omega^{\prime}} \mathscr{H} \square \omega_{\omega^{\prime \prime}}$ is 1-dimensional.

Proof. Let $\sigma_{\imath}$ denote the simple transposition of $i$ and $i+1$. If $i$ is a descent of $\omega^{\prime}$, i.e. $\omega_{i}^{\prime}>\omega_{i+1}^{\prime}$, then $\nabla_{\omega^{\prime}}=\nabla_{\omega^{\prime} \sigma_{t}} \nabla_{i}$, and

$$
\nabla_{\omega^{\prime}} T_{i}=\nabla_{\omega^{\prime} \sigma_{t}}\left(\nabla_{t} T_{i}\right)=-\nabla_{\omega^{\prime} \sigma_{l}} \nabla_{t}=-\nabla_{\omega^{\prime}}
$$

Thus, by induction, one obtains that the space $\nabla_{\omega^{\prime}} \mathscr{H}$ is linearly generated by the $\left\{\nabla_{\mu}, l(\mu)=l\left(\omega^{\prime}\right)+l\left(\omega^{\prime} \mu\right)\right\}$. These permutations $\mu$ are exactly the permutations having the decreasing subwords of $\omega^{\prime}$. Now, given such a $\mu$, suppose that $\mu_{\imath}>\mu_{t+1}$ and that $(i+1) i$ is a subword of $\omega^{\prime \prime}$ (i. e. that $\nabla_{l} \square_{\omega^{\prime \prime}}=0$ ). Then

$$
\nabla_{\mu}=\nabla_{\mu \sigma_{i}}\left(\nabla_{i}+q \frac{\left[\mu_{t}-\mu_{t+1}-1\right]}{\left[\mu_{i}-\mu_{\imath+1}\right]}\right)
$$

If $\mu \sigma_{i}$ has no longer the decreasing subwords of $\omega^{\prime}$, then $\mu_{i}-\mu_{i+1}=1$ and $\nabla_{\mu} \square \square_{\omega^{\prime \prime}}=$ $\nabla_{\mu \sigma_{t}}\left(\nabla_{i} \square \omega^{\prime \prime}\right)=0$. Otherwise $\nabla_{\mu} \square_{\omega^{\prime \prime}}$ is proportional to $\nabla_{\mu \sigma_{l}} \square_{\omega^{\prime \prime}}$. Therefore $\nabla_{\omega^{\prime}} \mathscr{H} \square_{\omega^{\prime \prime}}$ is generated by the elements $\nabla_{\mu} \square_{\omega^{\prime \prime}}$, where $\mu$ has the decreasing subwords of $\omega^{\prime}$ and is such that $\mu_{i}<\mu_{i+1}$ whenever $(i+1) i$ is a subword of $\omega^{\prime \prime}$. It turns out that there is only one permutation $\mu$ satisfying these two conditions. This permutation is the row reading of the Young tableau whose column reading is $\omega^{\prime}$. 
For example, if $I=331$, then $J=223, \omega^{\prime}=3216547, \omega^{\prime \prime}=2143765$. The only permutation $\mu$ having subwords $321,654,7$ and such that $\mu_{1} \ngtr \mu_{2}, \mu_{3} \ngtr \mu_{4}, \mu_{5} \ngtr$ $\mu_{6}, \mu_{6} \ngtr \mu_{7}$ is 3625147. This permutation is the row-reading of the Young tableau

$\begin{array}{lll}3 & 6 & \\ 2 & 5 & \\ 1 & 4 & 7\end{array}$

Similarly, the space $\square \omega_{\omega^{\prime \prime}} \mathscr{H} \nabla_{\omega^{\prime}}$ is 1 -dimensional and equal to $\square_{2471365} \nabla_{3216547}$. This time, one recognizes in the new permutation the row-reading of the contretableau

$$
\begin{array}{lll}
2 & 4 & 7 \\
1 & 3 & 6 \\
& & 5
\end{array}
$$

The preceding Lemma enables one to construct primitive idempotents of $\mathscr{H}_{n}$. More precisely we have

Theorem 5. 2. Let I be a partition, $\omega^{\prime}$ and $\omega^{\prime \prime}$ the maximal elements of the corresponding Young subgroups, $\mu^{\prime}$ and $\mu^{\prime \prime}$ the row readings of the associated tableau and contretableau. Then $\nabla_{\mu^{\prime}} \square \mu_{\mu^{\prime \prime}}$ and $\square_{\mu^{\prime \prime}} \nabla_{\mu^{\prime}}$ are two idempotents, up to a scalar, and their images are irreducible representations of the Hecke algebra corresponding to the partition I.

In the preceeding example, the claim is that $\nabla_{3625147} \square_{2471365}$ and $\square_{2471365} \nabla_{3625147}$ are two $q$-idempotents, up to a factor.

Using now our interpretation of $\mathscr{H}_{n}$ as an algebra of operators on the ring of polynomials, we can realize its irreducible representations by letting it act on an appropriate subspace of $\mathbb{K}[\mathbb{A}]$.

Let $I \in \mathbb{N}^{r}$ be an integral vector of weight $n, \mathscr{S}(I)$ be the corresponding Young subgroup of $\mathfrak{S}_{n}$, and $\omega_{I}$ its maximal element, $d$ the length of $\omega_{I}$. Let $j_{0}=$ $0, j_{1}=i_{1}, j_{2}=i_{1}+i_{2}, \ldots, j_{r}=i_{1}+\ldots+i_{r}$. The $q$-Vandermonde of index $I$ is by definition

$$
\Delta_{I}:=\prod_{m=0}^{r-1} \prod_{j m<k<l \leq j m+1}\left(q a_{l}-a_{k}\right) .
$$

Then, $\xi_{I}:=\square_{\nu} \nabla_{\omega_{I}}=\square_{\nu} \partial_{\omega_{I}} \Delta_{I}$ is the idempotent corresponding to $I$.

Since the operator $\square_{\nu} \partial_{\omega_{I}}$ decreases degrees by $d$, it sends the space $K[\mathbb{A}]^{d}$ of polynomials of degree $d$ to $K$ because it is not identically null on that space. Therefore the space $\mathbb{K}[\mathrm{A}]^{d} \xi_{I}$ is 1 -dimensional of basis $\Delta_{I}$ and the space $\Delta_{I} \mathscr{H}_{n}=K$ $[\mathrm{A}]^{d} \xi_{I} \mathscr{H}_{n}$, which is equal to $\mathscr{H}_{n} \xi_{I} \mathscr{H}_{n}$, is, according to theorem 5. 2, a model of an irreducible representation of $\mathscr{H}_{n}$, corresponding to the shape conjugate to the 
reordering of $I$ into a partition.

We shall explicit this representation when $I$ is weakly increasing, i. e. is a partition.

Let $t$ be the contretableau which, as a word, coincides with $\omega_{I}$. Consider the set of permutations

$$
\mathscr{P}_{\operatorname{erm}}(I)=\left\{\omega_{I}^{-1} w \mid w \equiv t\right\}
$$

for all $w$ plactically congruent ( $\equiv$ ) to $t$.

For example, with $I=122, \omega_{I}=13254$,

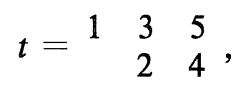

the plactic class of $t$ is the set $\{13254,31254,13524,31524,35124\}$ and

$$
\mathscr{P}_{\text {erm }}(I)=\{12345,21345,12435,21435,24135\}
$$

Instead of the class of $t$, one can use Yamanouchi symbols (cf. [Ham]) (called lattice permutations by MacMahon, see [Mcd]) : one starts with the code of $\omega_{I}$, i. e. with the vector $\left[i_{1}-1, \ldots, 1,0, i_{2}-1, \ldots, 1,0, \ldots, i_{r}-1, \ldots, 1,0\right]$; its plactic class is the set $\mathscr{Y}_{\operatorname{am}}(I)$ of Yamanouchi symbols corresponding to shape $I$. For example, the code of $\omega_{122}=13254$ is $[0,1,0,1,0]$ and its class $\mathscr{Y}_{a m}(122)$ is

$$
\{[0,1,0,1,0],[1,0,0,1,0],[0,1,1,0,0],[1,0,1,0,0],[1,1,0,0,0]\}
$$

which coincides with the orbit of the code of $\omega_{122}$ under the set of permutations $\mathscr{P}_{\text {erm }}(122)$.

It is casy to characterize the set of all Yamanouchi symbols : they are exactly the vectors $v$ such that for any cut into two pieces : $v=v^{\prime} v^{\prime \prime}$, then the right vector $v^{\prime \prime}$ is such that

$$
\left|v^{\prime \prime}\right|_{0} \geq\left|v^{\prime \prime}\right|_{1} \geq\left|v^{\prime \prime}\right|_{2} \geq \cdots
$$

where $|v|_{i}$ denotes the number of components of a vector $v$ equal to $i$.

In the preceeding case, we have enumerated the vectors permuted from $[0,1$, $0,1,0]$ which have more 0 than 1 in any of their right part, excluding the five vectors $\{[0,0,0,1,1],[0,0,1,0,1],[0,1,0,0,1],,[1,0,0,0,1],[0,0,1,1,0]\}$. Note that Yamanouchi symbols are another way of coding standard Young tableaux.

We now claim that Yamanouchi symbols are the exponents of the leading (for the lexicographic order) monomials of the polynomials $\left\{\Delta_{I} T_{\mu}: \mu \in \mathscr{P}_{\text {erm }}(I)\right\}$. The case $I=122$ will be sufficient to illustrate this property. The polynomial 


$$
\Delta_{I}=e^{01010}-q e^{00110}-q e^{01001}+q^{2} e^{00101}
$$

has only one monomial whose exponent satisfies the Yamanouchi condition, that is $e^{01010}$. The successive images of this monomial under the $T_{\mu}$ are the polynomials $e^{10010}, e^{01100}, e^{10100}, e^{11000}$, i. e. the monomials having a Yamanouchi exponent, and the other monomials in the expansions of $\Delta_{I} T_{\mu}$ are lexicographicaly smaller than $e^{01010} T_{\mu}$.

Therefore, the polynomials $\Delta_{I} T_{\mu}$ are linearly independent, and constitute a basis because their number is equal to the number of Young tableaux of shape $I$. Moreover, the projection of $\Delta_{I} \mathscr{H}_{n}$ on the free module of basis $\left\{e^{y}, y \in \mathscr{Y}\right.$ am $\left.(\mathbb{I})\right\}$ is an isomorphism.

Let $M$ be the matrix whose columns are the coefficients of the Yamanouchi monomials of the polynomials $\Delta_{I} T_{\mu}$. Then $M$ is a triangular matrix with unit diagonal. For $q=1$, this matrix, which has only $0, \pm 1$ entries, has been defined by Rutherford to describe the so-called Young natural representation. It is interpreted in $[\mathrm{CLL}]$ as a matrix of scalar products and used to produce a turbo-straightening for decompositions into standard bases.

From what precedes, the matrix $M$ also gives decompositions into standard bases in the case of Hecke algebras. Let $h$ belong to the irreducible subspace $\xi_{I} \mathscr{H}_{n}$. To decompose it into the basis $\xi_{I} \mathbb{T}_{\mu}$, one must compute the image of the leading monomial in $\Delta_{I}$ (which is the only one having a Yamanouchi symbol as exponent) under $h$, restrict it to its Yamanouchi terms, and take the image under the inverse of the matrix $\mathbb{M}$.

In other words, one has the following proposition.

Proposition 5. 3. Given a partition I of $n, \Delta_{I} \mathscr{H}_{n}$ is an irreducible representation of the Hecke algebra $\mathscr{H}_{n}$, with basis $\left\{\Delta_{I} \mathbb{T}_{\mu}, \mu \in \mathscr{P}_{\text {erm }}(I)\right\}$. The projection on the space generated by the $\left\{e^{y}, y \in \mathscr{Y}\right.$ am $\left.(I)\right\}$ induces an isomorphism of vector spaces between $\Delta_{I} \mathscr{H}_{n}$ and $\left\{e^{y}, y \in \mathscr{Y}\right.$ am $\left.(\mathbb{I})\right\}$. The matrix expressing the images of the basis $\left\{\Delta_{I} T_{\mu}\right\}$ is triangular with unit diagonal.

For example, in the above case $I=122$, the base of polynomials $\left\{\Delta_{I} T_{\mu}, \mu \in\right.$ $\mathscr{P}$ erm $(\mathbb{I})\}$ is

$$
\begin{aligned}
& \Delta_{122}=[01000]-q[00110]-q[01001]+q^{2}[00101] \\
& \Delta_{122} T_{1}=[10010]-q[10001]-q^{2}[00110]+q^{3}[00101] \\
& \Delta_{122} T_{3}=[01100]-q^{2}[01001]-q^{2}[00110]+\left(q^{3}-q^{2}\right)[00101]+q^{3}[00011] \\
& \Delta_{122} T_{1} T_{3}=[10100]-q^{2}[10001]-q^{3}[00110]+\left(q^{4}-q^{3}\right)[00101]+q^{4}[00011] \\
& \Delta_{122} T_{1} \mathbb{T}_{3} T_{2}=[11000]-q^{3}[10001]-q^{3}[01010]+\left(q^{4}-q^{3}\right)[01001]+q^{5} \\
& {[00011]}
\end{aligned}
$$


Therefore, the projection of this basis onto the space $\left\{e^{y}, y \in \mathscr{Y} a m(122)\right\}$ is represented by the matrix

$$
\left(\begin{array}{lllll}
1 & 0 & 0 & 0 & q^{3} \\
0 & 1 & 0 & 0 & 0 \\
0 & 0 & 1 & 0 & 0 \\
0 & 0 & 0 & 1 & 0 \\
0 & 0 & 0 & 0 & 1
\end{array}\right)
$$

\section{§ 6. Hook Representations}

Hook representations could be easily described in the framework of the preceding section and the next theorem could be directly checked on the explicit matrices of representation obtained above. There is, however, an alternative approach involving a $q$-analog of an interesting property of hook representations of the symmetric group.

Let $V=\mathbf{C} x_{1} \oplus \cdots \oplus \mathbf{C} x_{n}$ be the vector space of polynomials of degree 1 , and let $g_{\imath}$ be the restriction of the operator $T_{l}$ to $V$, so that

$$
x_{J} g_{l}=\left\{\begin{array}{cl}
q x_{J} & (j \neq i, i+1) \\
(q-1) x_{\imath}+q x_{\imath+1} & (j=i) \\
x_{\imath} & (j=i+1)
\end{array}\right.
$$

A basis of the space $\wedge^{k} V$ is given by the $x_{i_{1}} \wedge \cdots \wedge x_{i_{k}}\left(i_{1}<\ldots<i_{k}\right)$, and one can code such an element by a vector in $\{0,1\}^{n}$, the components equal to 1 being the $i_{1}, \ldots, i_{k}$-th ones.

The action of $\wedge^{k} g_{r}$ on these vectors is, writing only the $r$ and $r+1$-th components

$$
\begin{aligned}
& {[\cdots 00 \cdots] \longrightarrow q^{k}[\cdots 00 \cdots]} \\
& {[\cdots 01 \cdots] \longrightarrow q^{k-1}[\cdots 10 \cdots]} \\
& {[\cdots 10 \cdots] \longrightarrow q^{k-1}(q-1)[\cdots 10 \cdots]+q^{k}[\cdots 01 \cdots]} \\
& {[\cdots 11 \cdots] \longrightarrow q^{k-1}[\cdots 11 \cdots]}
\end{aligned}
$$

It is immediate to check on relations (5. 1) that the operators $g_{i}^{(k)}=q^{1-k} \wedge^{k} g_{\imath}$ satisfy the Hecke relations, so that $\eta_{k}: T_{i} \longrightarrow g_{i}{ }^{(k)}$ defines a representation of $\mathscr{H}_{n}$ in $\wedge^{k} V$.

The irreducible content of this representation can be identified by a standard specialization argument, which can be conveniently formulated as follows. To any representation $\rho$ of the Hecke algebra, it is possible to associate a symmetric function $F=\mathscr{F}(\rho)$ which depends only of the traces of the representation matrices of certain elements. The result ( $c f .[\mathrm{KW}],[\mathrm{Ram}])$ is that this symmetric function is independent of $q$ and is equal to the Frobenius characteristic of the representation of the symmetric group obtained by setting $q=1$. We shall also call $\mathscr{F}(\rho)$ the 
Frobenius characteristic of $\rho$.

In the case of $\eta_{k}$, the specialization $q=1$ yields the $k$-th exterior power of the representation of $\mathfrak{S}_{n}$ by permutation matrices, whose Frobenius characteristic is well known to be equal to $\Lambda_{k} S_{n-k}$ [Ait], where $\Lambda_{k}$ is the $k$-th elementary symmetric function, and $S_{n-k}$ a complete symmetric function (defined by $\sum_{j} z^{j} S_{j}(X)=\Pi_{x \in X}$ $\left.(1-z x)^{-1}\right)$.

Moreover, the same specialization argument shows that $V$ splits into two irreducible components as an $\mathscr{H}_{n}$-module

$$
V=V^{(1, n-1)} \oplus V^{(n)}
$$

where $V^{I}$ affords an irreducible representation indexed by the partition $I$. Let $\left(u_{1}\right.$, $\left.\ldots, u_{n}\right)$ be a basis of $V$ formed by taking a basis $\left(u_{1}, \ldots, u_{n-1}\right)$ of $V^{(n-1)}$ and a basis $\left(u_{n}\right)$ of $V^{(n)}$.

Then, the restrictions of the $g_{i}^{(k)}$ to the subspace

$$
W_{k}=\operatorname{vect}\left\{u_{i_{1}} \wedge \cdots \wedge u_{i_{k}} \mid 1 \leq i_{1}<i_{2}<\ldots<i_{k} \leq n-1\right\}
$$

define a representation $\eta_{k}^{\prime}$ of $\mathscr{H}_{n}$, which for $q=1$ reduces to the $k$-th exterior power of the fundamental representation $(1, n-1)$ of $\mathfrak{S}_{n}$. This exterior power is known to have as Frobenius characteristic the hook Schur function $S_{1^{k}, n-k}$, whence the following result, obtained by Jones ([Jo], p. 354).

Proposition 6. 1. Let $V^{(1, n-1)}$ be the fundamental representation of dimension $n-1$ of the Hecke algebra $\mathscr{H}_{n}$, and $M_{1}, M_{2}, \ldots, M_{n-1}$ be the matrices representing the generators of $\mathscr{H}_{n}$. Then, the matrices $q^{1-k} \wedge^{k} M_{i}$ generate an irreducible representation of $\mathscr{H}_{n}$, corresponding to the hook $\left(1^{k}, n-k\right)$.

Taking into account the generating function of the values of the hook characters $[\mathrm{KW}]$, $[\mathrm{Ram}]$, we arrive at the following identity : denoting by $\rho$ the fundamental representation indexed by the hook $(1, n-1)$, the characteristic polynomial of an element $T_{\mu}$ of connectivity class corresponding to the partition $I$ $=\left(1^{m_{1}} 2^{m_{2}} \cdots n^{m_{n}}\right)($ see $[\mathrm{KW}])$ is given by

$$
q^{l(\mu)} \operatorname{det}\left(I d-\frac{z}{q^{l(\mu)}} \rho\left(T_{\mu}\right)\right)=(1-z)^{l(I)-1} \prod_{k=1}^{n}\left(\frac{q^{k}-z^{k}}{q-z}\right)^{m_{k}}
$$

In particular, this implies that the eigenvalues of the $T_{\mu}$ in the fundamental representation, and then also in all hook representations, are roots of unity multiplied by powers of $q$. 


\section{§ 7. Kazhdan-Lusztig Representations}

Kazhdan and Lusztig have defined a linear basis $\left\{c_{\mu}, \mu \in \mathbb{S}_{n}\right\}$ of the Hecke algebra $\mathscr{H}_{n}$, by some optimal properties $[\mathrm{KL}]$. Subsets of these bases give representations of $\mathscr{H}_{n}$, irreducible representations arising as factors of these representations. Instead of taking quotient representations, $K \& \mathrm{~L}$ describe for each partition $I$ a graph, from which one can read the matrices representing the generators $T_{1}, \ldots, T_{n-1}$ in the irreducible representation of index $I$.

The vertices of this graph are as usual all the standard Young tableaux of shape $I$ (left cell) or the elements of a plactic class (right cell). Unfortunately, the edges are not easy to obtain in general, being related to the expansion of the $c_{\mu}$ into the basis $\left\{T_{\mu}\right\}$. More precisely, there is an edge or not whether or not the coefficients in this expansion (Kazhdan-Lusztig Polynomials) are of maximal degree. However, since it is difficult to compute these polynomials, the Kazhdan-Lusztig description of representations is difficult to put into concrete use.

Let us recall how Kazhdan and Lusztig code the representation matrices of the generators of $\mathscr{H}_{n}$ by a graph. To a set of permutations $\left\{v, v^{\prime}, \ldots\right\}$ one associates a labelled graph. The set of vertices is $\left\{v, v^{\prime}, \ldots\right\}$; there is an edge $v-v^{\prime}$ iff $v$ and $v^{\prime}$ differ by a simple transposition. Each vertex $v$ is endowed with a set of integral labels $\mathscr{L}(v)$ defined as follows : put label $i$ iff the vertex has a subword $(i+1 i)$. Then the matrix $M_{i}$ has a non-zero entry $\left(=q^{l\left(v^{\prime}\right)-l(v)}\right)$ corresponding to the pair $v$, $v^{\prime} v \neq v^{\prime}$, iff $v^{\prime}$ has label $i$ and $v$ has not. The entry $v, v$ is equal to -1 or $q$ whether $v$ has label $i$ or not.

In the case of a hook partition, however, one has explicit graphs. Indeed, fix $k, n$ and let $\omega$ be the permutation $\omega=(1 \ldots, n-k-1, n, \ldots, n-k)$, and let $\mu$ be any element in the plactic class of $\omega$. One uses the following criterium of "non-singularity" :

Theorem 7. 1 [Lak-Sa $]$. Let $\mu$ be a permutation. Then the following conditions are equivalent

i) the Schubert variety of index $\mu$ in the flag manifold is non singular

ii) the Kazhdan-Lusztig element $c_{\mu}$ is equal to $\sum_{\nu \leq \mu}(-q)^{l(\mu)-l(\nu)} T_{\nu}$, the order on permutations being the Ehresmann/Bruhat order.

iii) $\mu$, considered as a word, does not contain subwords of the type hkij or kjhi, for $i<j<h<k$.

Now it is clear from the description that we gave of the class of $\omega$ that any $\mu$ in this class satisfy [Lak-Sa] third condition. Therefore, all associated KazhdanLusztig polynomials are trivial, which implies that for a hook, there is an edge connecting two elements iff they differ by a simple transposition.

For example, for the hook (114), one has the graph 


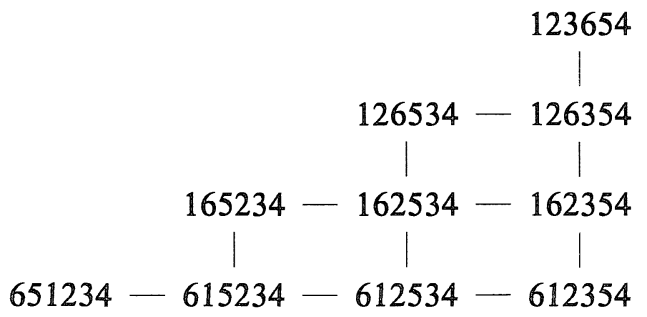

It is in fact possible to write explicitely the $\mathbb{K a z h d a n}-\mathbb{L}$ usztig element $c_{\mu}$ for any non-singular $\mu$ : it is a product of simple factors of the type $\left(\nabla_{i}+q \frac{[r-1]}{[r]}\right)\left(\right.$ the $c_{\mu}$ do not coincide with the $\nabla_{\mu}$, though they both factorize).

For example, for the hook 112 , one has

$$
\left\{c_{1243}=\nabla_{3}, c_{1423}=\nabla_{3} \nabla_{2}, c_{4123}=\nabla_{3} \nabla_{2} \nabla_{1}\right\}
$$

but

$$
\begin{gathered}
\left\{\nabla_{1243}=\nabla_{3}, \nabla_{1423}=\nabla_{3}\left(\nabla_{2}+\frac{q}{1+q}\right)\right. \\
\left.\nabla_{4123}=\nabla_{3}\left(\nabla_{2}+\frac{q}{1+q}\right)\left(\nabla_{1}+\frac{q+q^{2}}{1+q+q^{2}}\right)\right\} .
\end{gathered}
$$

Now one can compute the $c_{\mu} \mathbb{T}_{l}$, but if one sticks to the Kazhdan-Lusztig approach, one has to take the quotient modulo the components of other types. It is simpler, instead of taking quotients, to use the interpretation of the elements of $\mathscr{H}_{n}$ as operators and restrict their action to an appropriate subspace of polynomials so that the extra components vanish.

In fact, one can avoid new computations and interpret the results of the preceding sections in terms of a graph, in which we shall recognize a Kazhdan-Lusztig graph. Let us first consider the case of the hook $(1, n-1)$. The underlying space is the space of polynomials of degree 1 modulo the ideal generated by symmetric polynomials without constant term, with basis the Schubert polynomials. In other words, we take the space $\mathscr{V}_{1}$ of polynomials of degree 1 , with basis $\left\{a_{1}+\cdots+a_{n-1}, \ldots, a_{1}+a_{2}, a_{1}\right\}$ and relation $a_{1}+\cdots+a_{n}=0$.

Each $T_{1}$ acts trivially (i.e. by multiplication by $q$ ) on Schubert polynomials (because they are symmetrical in $a_{i}, a_{i+1}$, except for $a_{1}+\cdots+a_{i}$. The representing matrix of $T_{i}$ coincide with $q \times$ identity, except for its $(n-i)$-th column which is

$$
[0, \ldots, 0, q,-1,1,0, \ldots, 0]
$$

the -1 being on the diagonal. 
These are the same matrices as the Kazhdan-Lusztig ones. Section 6 shows that one can take any exterior power of this representation and finally one obtains :

Proposition 7. 2. Given two integers $h<k$, the labelled graph with vertices the elements of the plactic class of $(1,2, \ldots, n-k-1, n, \ldots, n-k)$, and with edges corresponding to each pair of vertices differing by a simple transposition, codes an irreducible representation, corresponding to the hook $\left(1^{k}, n-k\right)$.

\section{§ 8. Application to a Quantum Spin Chain Model}

As shown by Deguchi and Akutsu [DA], the exact solution of a whole class of quantum spin chain models can be reduced to the diagonalization of the images of the following element of the Hecke algebra

$$
H=\sum_{i=1}^{n-1}\left(T_{\imath}-q\right)=\sum_{i=1}^{n-1} \nabla_{l}
$$

under some set of irreducible representations, depending on the symmetry algebra of the chain (the Hamiltonian being the image of $H$ under some representation of $\left.\mathscr{H}_{n}\right)$.

For example, the model having the quantum superalgebra $U_{q}(\mathfrak{s u}(1 / 1))$ as symmetry algebra leads to the family of hook representations (see e. g. [MR] and references therein). In this case, the hamiltonian can be diagonalized by means of a Jordan-Wigner transformation $[\mathrm{HR}]$. However, the results of the preceding two sections lead to an alternative approach, which is perhaps more illuminating.

Using as in Section 7 the basis of Schubert polynomials $e_{n-i}=a_{1}+a_{2}+\cdots+a_{i}$ $(1 \leq i \leq n-1)$ in the space of linear polynomials modulo $a_{1}+\cdots+a_{n}$, we see that the matrix of the image of $H$ in the representation $(1, n-1)$ can be written

$$
\rho_{(1, n-1)}(H)=A_{n-1}(q)-(1+q) I_{n-1}
$$

where $I_{n-1}$ is the identity matrix and

$$
A_{n-1}(q)=\left(\begin{array}{ccccccc}
0 & q & 0 & 0 & \cdots & 0 & 0 \\
1 & 0 & q & 0 & \cdots & 0 & 0 \\
0 & 1 & 0 & q & \cdots & 0 & 0 \\
\vdots & \vdots & \vdots & \vdots & & \vdots & \vdots \\
0 & 0 & 0 & 0 & \cdots & 1 & 0
\end{array}\right)
$$

Set $q=t^{2}$ and let $P_{n}(x, t)$ be the characteristic polynomial of $A_{n}(q)$. Then, $P_{2}(x, t)=x^{2}-t^{2}$ and one has the recurrence relation

$$
P_{n+1}(x, t)=-x P_{n}(x, t)-t^{2} P_{n-1}(x, t)
$$


which shows by induction that $P_{n}$ is homogeneous of degree $n$ in $x$ and $t$. Moreover, (8.1) also shows that for $t=1, P_{n}(x, 1)=U_{n}(x / 2)$, where $U_{n}$ is the Chebyschev polynomial of the second kind. The characteristic polynomial of $A_{n-1}(q)$ is thus

$$
q^{(n-1) / 2} U_{n-1}\left(\frac{x}{2 \sqrt{q}}\right)
$$

so that its eigenvalues are

$$
\lambda_{s}=2 \sqrt{q} \cos \left(\frac{s \pi}{n}\right)
$$

The components $v_{i}^{(s)}$ of an eigenvector $v^{(s)}$ associated to $\lambda_{s}$ satisfy the difference equation

$$
\lambda_{s} v_{i}^{(s)}=v_{i-1}^{(s)}+q v_{i+1}^{(s)}
$$

with the boundary conditions $v_{0}^{(s)}=v_{n}^{(s)}=0$, so that one can take

$$
v^{(s)}=\left(\begin{array}{c}
q^{-1 / 2} \sin (s \pi / n) \\
q^{-3 / 2} \sin (2 s \pi / n) \\
\vdots \\
q^{-(n-1) / 2} \sin ((n-1) s \pi / n)
\end{array}\right)
$$

Remark. The transition matrix from the basis $\left(e_{i}\right)$ to the basis $\left(v^{(j)}\right)$ can be written $B_{n-1}(q)=G_{n-1}(q) C_{n-1}$ where $G_{n-1}(q)=\operatorname{diag}\left(q^{-1 / 2}, q^{-3 / 2}, \ldots, q^{-(n-1) / 2}\right)$ and $C_{n-1}=B_{n-1}(1)$. One easily checks that $C_{n-1}^{2}=(n / 2) I$, so that the inverse of $B_{n-1}$ is also explicitely known.

The diagonalization of $H$ in any hook representation can now be deduced from the above considerations by means of the following observation. Given a vector space $V$ and an endomorphism $f$ of $V$, denote by $D_{f}$ the unique derivation of the exterior algebra $\Lambda(V)$ which coincides with $f$ on $V$. Taking for $V$ the space of linear polynomials and $f=g_{i}$ the restriction of $T_{i}$ to $V$ as in Section 6, we obtain by a direct and easy computation :

Lemma 8. 1. Let $D_{l}^{(k)}$ be the restriction of $D_{t}=D_{g_{t}}$ to $\wedge^{k} V$. Then,

$$
D_{i}^{(k)}=q^{1-k} \wedge^{k} g_{i}+(k-1) I
$$

Now, let $h$ be the restriction of $H$ to $V$, i.e. 


$$
h=\sum_{i=1}^{n-1}\left(g_{i}-q I\right)=\sum_{i=1}^{n-1} g_{i}-(n-1) q I .
$$

We have $D_{f+g}=D_{f}+D_{g}$ and $D_{i d_{V}}^{(k)}=k \cdot i d \wedge^{k} V=k$. I. Hence,

$$
\begin{aligned}
D_{h}^{(k)} & =\sum_{i=1}^{n-1} D_{i}^{(k)}-k(n-1) q \cdot I=\sum_{i=1}^{n-1}\left[q^{1-k} \wedge^{k} g_{i}-q I\right]-(k-1)(n-1) q I \\
& =\eta_{k}(H)-(k-1)(n-1) q I
\end{aligned}
$$

where $\eta_{k}$ is the representation of Frobenius characteristic $\Lambda_{k} S_{n-k}$ considered in Section 6. And as we saw in Section 6, the irreducible representation corresponding to the hook $\left(1^{k}, n-k\right)$ can be realized as a subrepresentation $\eta_{k}^{\prime}$ of $\eta_{k}$ : taking any basis $\left(u_{1}, \ldots, u_{n}\right)$ of $V$ such that $u_{1}, \ldots, u_{n-1}$ generate the irreducible component $V^{(1, n-1)}, u_{n}$ being a basis of $V^{(n)}$, we have

$$
V^{\left(1^{k}, n-k\right)}=\operatorname{vect}\left\{u_{i_{1}} \wedge \cdots \wedge u_{i_{k}} \mid i_{1}, \ldots, i_{k} \leq n-1\right\}
$$

Then, $\rho_{\left(1^{k}, n-k\right)}(H)=\eta_{k}^{\prime}(H)$ is equal to the restriction to $V^{\left(1^{k}, n-k\right)}$ of the operator $D_{H}^{(k)}+(k-1)(n-1) q I$. The eigenvalues of the restriction of $D_{H}^{(k)}$ to $V^{\left(1^{k}, n-k\right)}$ $=\wedge^{k} V^{(1, n-1)}$ are clearly the sums $k$ by $k$ of the eigenvalues of the restriction of $h$ to $V^{(1, n-1)}$. These eigenvalues are $\mu_{s}=\lambda_{s}-(1+q)$ so that we get the complete spectrum of $\rho_{\left(1^{k}, n-k\right)}(H)$ :

Theorem 8. 2. The eigenvalues of the hook hamiltonian $\rho_{\left(1^{k}, n-k\right)}(H)$ are exactly

$$
\lambda_{t_{1}}+\lambda_{t_{2}}+\cdots+\lambda_{t_{k}}+((k-1)(n-1)-k) q-k
$$

and the eigenvectors are explicitely given by the above construction.

Remark. Using the same kind of method, one can also obtain all the spectral parameters of $H_{k}=T_{1}+T_{3}+\ldots+T_{2 k+1}$ or equivalently of any sum of $k$ commuting generators of the Hecke algebra. Indeed it can be shown that the eigenvalues of $H_{k}$ are exactly

$$
\lambda_{\varepsilon}=\sum_{\imath=1}^{k} q^{\varepsilon_{\imath}} \varepsilon_{t}
$$

for every $\varepsilon=\left(\varepsilon_{1}, \varepsilon_{2}, \ldots, \varepsilon_{k}\right) \in\{-1,1\}^{k}$, the multiplicity of $\lambda_{\varepsilon}$ being the number of different ways it can be obtained in such a sum. The eigenvectors $v_{\varepsilon}$ of $H_{k}$ may also be explicitely described in a similar way as above.

Denoting by $P(k \mid z)$ the characteristic polynomial of $\rho_{\left(1^{k}, n-k\right)}(H)$, it follows 
from the last theorem that we have

$$
P(k \mid z)=q^{\frac{n-1}{2}} P_{n}^{(k)}\left(q^{-\frac{1}{2}}(z-((k-1)(n-1)-k) q-k)\right)
$$

where we set

$$
P_{n}^{(k)}(X)=\prod_{1 \leq j_{1}<j_{2}<\cdots j_{k} \leq n-1}\left(X-\left(\sum_{m=1}^{k} 2 \cos \left(\frac{j_{m} \pi}{n}\right)\right)\right)
$$

Since $\boldsymbol{P}_{n}^{(k)}$ is obtained by means of an integer translation from the characteristic polynomial of a graph, it lies in $\mathbb{Z}[X]$. We shall now describe its factorization in this ring.

The field $K_{n}=\mathbf{Q}\left[e^{\frac{i \pi}{n}}\right]$ is a splitting field for every $P_{n}^{(k)}$. The Galois group $\operatorname{Gal}\left(K_{n} \mid \mathbf{Q}\right)$ is isomorphic to the group $U$ of units of $\mathbf{Z} / 2 n \mathbf{Z}$, the correspondence being $k \in U \longrightarrow \sigma_{k} \in \operatorname{Gal}\left(\boldsymbol{K}_{i n} \mid \mathbf{Q}\right)$ where, for every $k \in U, \sigma_{k}$ denotes the Q-automorphism of $K_{n}$ that maps $e^{\frac{i \pi}{n}}$ onto $e^{\frac{i k \pi}{n}}$. We now construct an action of $U$ on the parameters $\left(j_{1}, j_{2}, \ldots, j_{k}\right)$ involved in (8.2). For every $1 \leq l<m \leq k$, let $W_{l}$, $W_{l, m}^{+}$and $W_{l, m}^{-}$be the sets of $k$-uples $\left(j_{1}, j_{2}, \ldots, j_{k}\right)$ in $(\mathbb{Z} / 2 n \mathbb{Z})^{k}$ that are respectively defined by the equations $j_{l}=0, j_{l}+j_{m}=0$ and $j_{l}-j_{m}=0$. Let then

$$
R=(\mathbb{Z} / 2 n \mathbf{Z})^{k}-\bigcup_{1 \leq l \leq k} W_{l}-\bigcup_{1 \leq l<m \leq k} W_{l, m}^{+}-\bigcup_{1 \leq l<m \leq k} W_{l, m}^{-} .
$$

The set $R$ is invariant under the action of $U$ defined by

$$
u .\left(j_{1}, j_{2}, \ldots, j_{k}\right)=\left(u j_{1}, u j_{2}, \ldots, u j_{k}\right)
$$

for $u \in U$ and $\left(j_{1}, j_{2}, \ldots, j_{k}\right) \in R$. It is also invariant under the action of the hyperoctahedral group $B_{k}$ defined by

$$
(\varepsilon, \sigma) .\left(j_{1}, j_{2}, \cdots j_{k}\right)=\left(\varepsilon_{1} j_{\sigma(1)}, \varepsilon_{2} j_{\sigma(2)}, \ldots, \varepsilon_{k} j_{\sigma(k)}\right)
$$

for $(\varepsilon, \sigma) \in B_{k}$ and $\left(j_{1}, j_{2}, \ldots, j_{k}\right) \in R$ where $\varepsilon=\left(\varepsilon_{1}, \varepsilon_{2}, \ldots, \varepsilon_{k}\right) \in\{-1,1\}^{k}$ and $\sigma \in \mathfrak{S}_{k}$. It is straightforward to check that the simplex

$$
\Delta_{n}^{(k)}=\left\{\left(j_{1}, j_{2}, \cdots j_{k}\right) \in(\mathbb{Z} / 2 n \mathbb{Z})^{k} \mid 1 \leq j_{1}<j_{2}<\cdots j_{k} \leq n\right\}
$$

is a fundamental domain for this last action and that this action commutes with that of $U$. Let us consider the set $\Omega$ of orbits in $\Delta_{n}^{(k)}$ for the action defined by pulling back the action of $U$ on the quotient space $R / B_{k}$. We can now use $\Delta_{n}^{(k)}$ as a labelling for the roots of $\boldsymbol{P}_{n}^{(k)}$ with

$$
s\left(j_{1}, j_{2}, \cdots j_{k}\right)=\sum_{m=1}^{k} 2 \cos \left(\frac{j_{m} \pi}{n}\right) .
$$


Galois theory tells us then that, for every $\omega \in \Omega$ the polynomial

$$
P_{n, \omega}^{(k)}(X)=\prod_{l \in \omega}(X-s(l))
$$

lies in $\mathbf{Q}[\boldsymbol{X}]$ and that it is a primary polynomial ${ }^{(1)}$ on $\mathbf{Q}[\boldsymbol{X}]$. But one has

$$
P_{n}^{(k)}=\prod_{\omega \in \Omega} P_{n, \omega}^{(k)} .
$$

Thus, since $P_{n}^{(k)}$ is unitary, the factorization (8.3) lies in fact in $\mathbf{Z}[X]$. This explains the numerous factors of $\boldsymbol{P}_{n}^{(k)}$ that are experimentally observed.

Examples. Let us describe an example of factorization for $P_{n}^{(k)}$ that may be constructed by the previous method. Consider for instance the shape 1114 and the polynomial $P_{7}^{(3)}$. The elements of $U$ are here the units of $Z / 14 Z$ and hence are equal to $\{1,3,5,9,11,13\}$. Since 3 is a primitive element of $U$, we can describe the orbits of $\Omega$ under the action of $U$ by only looking on the iterated action of 3 on the elements of $\Delta_{7}^{(3)}$. Hence the orbit of $(1,2,3)$ under the previous action is given by

\begin{tabular}{|c|c|c|c|}
\hline Shape & $(n, k)$ & Orbits & Factors \\
\hline \multirow[t]{2}{*}{14} & $(5,1)$ & (1), (3) & $X^{2}+X-1$ \\
\hline & & (2), (4) & $X^{2}-X-1$ \\
\hline \multirow[t]{7}{*}{114} & $(6,2)$ & $(1,2),(2,5)$ & $X^{2}-2 X-2$ \\
\hline & & $(1,3),(3,5)$ & $X^{2}-3$ \\
\hline & & $(1,4),(4,5)$ & $X^{2}+2 X-2$ \\
\hline & & $(1,5)$ & $X$ \\
\hline & & $(2,3)$ & $X-1$ \\
\hline & & $(2,4)$ & $X$ \\
\hline & & $(3,4)$ & $X+1$ \\
\hline \multirow[t]{8}{*}{1114} & $(7,3)$ & $(1,2,3),(1,4,5),(3,5,6)$ & $X^{3}-X^{2}-9 X+1$ \\
\hline & & $(1,2,4),(2,3,6),(4,5,6)$ & $X^{3}+X^{2}-9 X-1$ \\
\hline & & $(1,2,5),(1,3,6),(3,4,5)$ & $X^{3}-X^{2}-2 X+1$ \\
\hline & & $(1,2,6),(3,4,6),(2,4,5)$ & $X^{3}+X^{2}-2 X-1$ \\
\hline & & $(1,3,4),(2,3,5),(1,5,6)$ & $X^{3}-X^{2}-2 X+1$ \\
\hline & & $(1,3,5)$ & $X-1$ \\
\hline & & $(2,3,4),(2,5,6),(1,4,6)$ & $X^{3}+X^{2}-2 X-1$ \\
\hline & & $(2,4,6)$ & $X+1$ \\
\hline
\end{tabular}

$$
\begin{aligned}
& (1,2,3) \longrightarrow 3 .(1,2,3)=(3,6,9)=(3,6,-5)=(3,5,6)\left[B_{3}\right] \in \Delta_{7}^{(3)}, \\
& (3,5,6) \longrightarrow 3 .(3,5,6)=(9,15,18)=(-5,1,4)=(1,4,5)\left[B_{3}\right] \in \Delta_{7}^{(3)}, \\
& (1,4,5) \longrightarrow 3 .(1,4,5)=(3,12,15)=(3,-2,1)=(1,2,3)\left[B_{3}\right] \in \Delta_{7}^{(3)}
\end{aligned}
$$

(1) More precisely $P_{n, \omega}^{(k)}=Q^{m}$ for some irreducible polynomial $Q$ and $m=\left|s^{-1}(s(l))\right|$ for any $l \in \omega$. 
Thus the desired orbit of $(1,2,3)$ is $\omega=\{(1,2,3),(1,4,5),(3,5,6)\}$ and the corresponding factor $\boldsymbol{P}_{7, \omega}^{(3)}$ of $\boldsymbol{P}_{\gamma}^{(3)}$ is then equal to

$$
\begin{gathered}
\left(X-\left(\xi+\xi^{13}+\xi^{2}+\xi^{12}+\xi^{3}+\xi^{11}\right)\right)\left(X-\left(\xi+\xi^{13}+\xi^{4}+\xi^{10}+\xi^{5}+\xi^{9}\right)\right) \\
\left(X-\left(\xi^{3}+\xi^{11}+\xi^{5}+\xi^{9}+\xi^{6}+\xi^{8}\right)\right)=X^{3}-X^{2}-9 X+1,
\end{gathered}
$$

where we set $\xi=e^{\frac{i \pi}{7}}$. More generally the tables in the previous page give some complete list of orbits of $\Omega$ with the corresponding factors of $\mathbb{P}_{n}^{(k)}$.

\section{§9. Conclusion}

A realisation of the Hecke algebra of type $A_{n}$ by symmetrization operators originated from algebraic geometry has been described. This point of view provides a convenient method for proving identities, as well as an explicit description of the irreducible representations. In particular, a fast "straightening" algorithm for the computation of representation matrices has been obtained. Different descriptions of hook representations have been presented, and illustrated on a quantum spin chain model. Other algebras of interest can also be realized by symmetrization operators, for example affine Hecke algebras (degenerate or not) and TemperleyLieb algebras. Also, identities between quantum determinants $[\mathbb{K L}]$ can be interpreted in the Hecke algebra, and symmetrization operators provide an alternative technique for proving them.

\section{Acknowledgements}

One of the authors (A. L.) warmly thanks Professors M. Kashiwara and T. Miwa for their invitation to visit RIMS during January 92.

\section{References}

[Ait] Aitken, A. C., On induced permutation matrices and the symmetric group, Proc. Edinburgh Math. Soc., (2) 5 (1936), 1-13.

[BGG] Bernstein, I. N., Gelfand, I. M. and Gelfand, S., Schubert cells and cohomology of the spaces G/P, Russian Math. Surveys, 28 (1973), 1-26.

[CLL] Carré, C., Lascoux, A. and Leclerc, B., Turbo-straigthening for decomposition into standard bases, Internat. J. Alg. Comp., 2 (1992), 275-290.

[Chel] Cherednik, I. V., On $R$-matrix quantization of formal loop groups, in Group theoretical methods in physics, Proc. 3rd Semin., Yurmala (USSR), 1985, 2 (1986), 161-180.

[Che2] - Quantum groups as hidden symmetries of classic representation theory, in Differential geometric methods in theoretical physics (A. I. Solomon, Ed), World Scientific, Singapore, 1989, pp. 47-54. 
[DA] Deguchi, T. and Akutsu, Y., Graded solutions of the Yang-Baxter relation and link polynomials, J. Phys. A, 23 (1990), 1861-1875.

[Dem] Demazure, M., Désingularisation des variétés de Schubert, Ann. Sci. Ec. Norm. Sup., 6 (1974), 53-88.

[DJ] Dipper, R. and James, G., Blocks and idempotents of Hecke algebras of general linear groups, Proc. London Math. Soc., 54 (1987), 57-82.

[FK1] Fomin, S. and Kirillov, A. N., The Yang-Baxter equation, symmetric functions and Schubert polynomials, preprint Inst. Mittag-Leffler (1992).

[FK2] - Yang-Baxter equation, symmetric functions and Grothendieck polynomials, preprint (1993).

[Gro] Grothendieck, A., Théorème de Riemann-Roch, in Séminaire de Géométrie Algébrique 6, Springer Lecture Notes in Math., 225 (1971).

[Gy] Gyoja, A., A q-analogue of Young symmetrizer, Osaka J. Math., 23 (1986), 841-852.

[Ham] Hamermesh, M., Group theory and its applications to physical problems, Addison-Wesley, Reading Mass., 1962.

[Hi1] Hirzebruch, F., Topological methods in algebraic geometry, Springer, Berlin, 1966.

[Hi2] Hirzebruch, F., Berger, T. and Jung, R., Manifolds and Modular Forms, Vieweg, Wiesbaden, (1992).

[HR] Hinrichsen, H. and Rittenberg, V., A two parameter deformation of the $S U(1 / 1)$ superalgebra and the $X Y$ quantum chain in a magnetic field, preprint CERN-TH. 6299/91, 1991.

[JK] James, G. D. and Kerber, A., The Representation Theory of the Symmetric Group, Addison-Wesley, Reading Mass., 1981.

[Jo] Jones, V., Hecke algebra representations of braid groups and link polynomials, Ann. of Math., 126 (1987), 335-388.

[KW] King, R. C. and Wybourne, B. G., Representations and traces of the Hecke algebras $H_{n}(q)$ of type $A_{n-1}, J$. Math. Phys., 33 (1992), 4-14.

[Lak-Sa] Lakshmibai, V. and Sheshadri, C. S., Singular locus of a Schubert variety, Bull. Amer. Math. Soc., (III) 2 (1984), 363-366.

[Las] Lascoux, A., Caractéristique d'Euler-Poincaré et produit des caractères, C. $R$. Acad. Sci. Paris Ser. I, 299 (1984), 447-450.

[LS82] Lascoux, A. and Schützenberger, M. P., Polynômes de Schubert, C. R. Acad. Sci. Paris, 294 (1982), 447-450.

[LS87] - Symmetrization operators on polynomial rings, Funct. Anal. Appl., 21 (1987), 77-78.

[Mcd] Macdonald, I. G., Symmetric functions and Hall polynomials, Oxford Math. Monographs, Clarendon Press, Oxford, 1979.

[Ma1] Martin, P., Potts models and related problems in statistical mechanics, World Scientific, Singapore, 1991.

[Ma2] The structure of $n$ variables polynomial rings as Hecke algebra modules, $J$. Phys. A, 26 (1993), 7311-7324.

[MR] Martin, P. and Rittenberg, V., A template for quantum spin chain spectra, Preprint RIMS Kyoto (1991).

[Ram] Ram, A., A Frobenius formula for the characters of Hecke algebras, Invent. Math., 106 (1991), 461-488.

[Ro] Rogawski, J. D., On modules over the Hecke algebra of a p-adic group, Invent. Math., 79 (1985), 443-465. 
